Proceedings

\title{
Commercially Fabricated Printed Circuit Board Sensing Electrodes for Biomarker Electrochemical Detection: The Importance of Electrode Surface Characteristics in Sensor Performance ${ }^{+}$
}

\author{
Gorachand Dutta ${ }^{1}$, Anna Regoutz ${ }^{2}$ and Despina Moschou 1,* \\ 1 Centre for Biosensors, Bioelectronics and Biodevices (C3Bio), Department of Electronic \& Electrical \\ Engineering, University of Bath, Bath BA2 7AY, UK; g.dutta@bath.ac.uk \\ 2 Department of Materials, Imperial College London, Exhibition Road, London SW7 2AZ, UK; \\ a.regoutz@imperial.ac.uk \\ * Correspondence: d.moschou@bath.ac.uk; Tel.: +44-(0)-1225-383245 \\ † Presented at the Eurosensors 2018 Conference, Graz, Austria, 9-12 September 2018. \\ Published: 29 November 2018
}

\begin{abstract}
Here we report the first PCB-implemented electrochemical glucose biosensor using covalently immobilized glucose oxidase (GOx) on the commercially fabricated PCB electrode surface, taking particular care on the electrode surface characteristics and their effect on sensor performance. Based on the results, this assay exhibits a highly linear response from $500 \mu \mathrm{M}$ to 20 $\mathrm{mM}(\mathrm{R}=0.9961)$ and a lower limit of detection of $500 \mu \mathrm{M}$.
\end{abstract}

Keywords: electrode pretreatment; electrochemical biosensor; printed-circuit-board; glucose detection; chronoamperometry

\section{Introduction}

Diabetes mellitus is an important chronic disease affecting increasing population numbers [1]. The World Health Organization (WHO) has placed diabetes among the top 10 world diseases with highest mortality rates. Until now, diabetes has been considered a disease of predominantly rich nations; this is not the case any more. Diabetes prevalence has been rising more rapidly in low and middle income countries; for example in Turkey prevalence stands at 12.8\% [2] (almost double the global average). While blood glucose diagnostic systems at the Point of Care are a world-wide success story, all the painless solutions (i.e., not requiring finger pricking to extract blood) available are too expensive (ca. $£ 60$ per sensor) to be considered as disposable diagnostics suitable for a mass population preventative screening in low and middle income countries. Hence the motivation of our work has been to develop a cost-effective glucose quantification microsystem, integrating sample delivery microfluidics and sensitive electrochemical biosensors on a seamlessly and commercially fabricated Lab-on-PCB platform [3]. In this work we focused on the development of a sensitive and reliable glucose biosensor, exploiting for the first time covalent immobilization of commercially fabricated PCB electrodes.

While several PCB-implemented electrochemical biosensors are being increasingly demonstrated over the past few years for several applications [4-9], little care is being given to the sensing electrode characteristics and their significance in sensor characteristics. Nonetheless, in the case of sensors exploiting SAM-assisted capture molecule immobilization, the physical and chemical properties of the electrodes can severely affect the sensing characteristics. Hence, in this paper we present the electrochemical and surface characteristics of the sensing electrodes as received by the 
manufacturer and also after our surface treatment process. Glucose oxidase is subsequently covalently immobilized on the chemically pre-treated, Au plated working electrodes, as opposed to previously presented PCB-implemented glucose sensors [10-13], resulting in a lower limit of detection of $500 \mu \mathrm{M}$.

\section{Materials and Methods}

Ammonium hydroxide, hydrogen peroxide, 3-Mercaptopropoinic acid, glucose oxidase, glucose and other reagents for buffer solutions were purchased from Sigma-Aldrich (Gillingham, UK). The sensing electrodes were designed in PCB CAD software (Altium ${ }^{\circledR}$, version 17.1.9) and commercially fabricated in a standard PCB manufacturing facility. The copper electrodes were electroplated with a hard-gold finish in order to exploit the pore-free deposition and low contact resistance achieved by this technique. The gold-plated electrodes were exploited as working, counter and reference electrodes and connected to a potentiostat to obtain the cyclic voltammograms and chronoamperometric signals. Glucose solution was serially diluted in PBS and used for electrochemical measurements. 50 $\mu \mathrm{L}$ of PBS sample was dispensed onto the sensor and cyclic voltammetric and chronoamperometric measurements were performed using an electrochemical workstation (Autolab, Utrecht, The Netherlands). Chronoamperometric signals were obtained at a bias potential of $0.2 \mathrm{~V}$.

\section{Results and Discussion}

The gold-plated electrodes were cleaned prior to GOx immobilization by 15 min ultrasonication in acetone, ethanol, and water, respectively and followed by $30 \mathrm{~min}$ ultrasonication in a solution containing 5:1:1 water, ammonium hydroxide $(20 \%)$ and hydrogen peroxide $(30 \%)$. Actually, the gold-plated electrodes are fully electrochemically inactive before cleaning (BC) as showed no redox peak in the cyclic voltammogram of $\mathrm{K}_{3} \mathrm{Fe}(\mathrm{CN})_{6}$ solution. Interestingly, after cleaning $(\mathrm{AC})$ process the electrodes were highly electrochemically active and showed a clear redox peak in the cyclic voltammogram of $\mathrm{K}_{3} \mathrm{Fe}(\mathrm{CN})_{6}$ solution. (Figure 1a). Atomic force micrographs (AFM) of Au plate electrodes were obtained before and after the cleaning (Figure 1b,c). The root-mean-square (RMS) roughness was decreased after the cleaning, probably due to the removal of organic layer from the Au surface. X-ray photoelectron spectroscopy (XPS) of all major core levels confirmed that the Au surface was initially covered under an organic layer of predominantly $\mathrm{C}$ and $\mathrm{O}$, which was removed almost completely after after the cleaning process (Figure 2). In addition, a strong $\mathrm{Cu}$ peak is found, giving a ratio of $\mathrm{Au}: \mathrm{Cu}=4: 1$. A self-assembled monolayer (SAM) of MPA (3-Mercaptopropoinic acid) was formed on the precleaned PCB gold electrode surface by immersing in an ethanol solution containing $1 \mathrm{mM}$ MPA for $1 \mathrm{~h}$ at RT followed by thoroughly rinsing with ethanol and DI water respectively and drying with $\mathrm{N}_{2}$ gas (Scheme 1). $2 \mathrm{mM}$ EDC and $5 \mathrm{mM}$ NHS solution mixture was incubated for $50 \mathrm{~min}$ on the electrode surface at RT to activate the carboxylic acid group of SAM. The electrodes were then washed twice with PBS. $20 \mu \mathrm{L}$ of PBS containing $5 \mathrm{mg} / \mathrm{mL}$ GOx was dispensed onto the electrode, incubated for overnight at $4{ }^{\circ} \mathrm{C}$. The electrodes were then washed twice with PBS and stored at $4{ }^{\circ} \mathrm{C}$. Figure 3a shows the successful immobilization of GOx on the gold-plated PCB electrode surface. Samples were prepared by serially diluting glucose in PBS. $70 \mu \mathrm{L}$ of sample was injected followed by the application of a $0.05 \mathrm{~V}$ bias potential. Chronoamperometric response profiles from $500 \mu \mathrm{M}$ to $20 \mathrm{mM}$ are shown in Figure $3 \mathrm{~b}$. A calibration plot of the chronoamperometric current at $60 \mathrm{~s}$ is shown in Figure $3 \mathrm{c}$ where each data point represents three separate measurements using new electrodes. Based on these results, this assay exhibits a highly linear response from $500 \mu \mathrm{M}$ to 20 $\mathrm{mM}(\mathrm{R}=0.9961)$ and a lower limit of detection of $500 \mu \mathrm{M}$. 
(a)

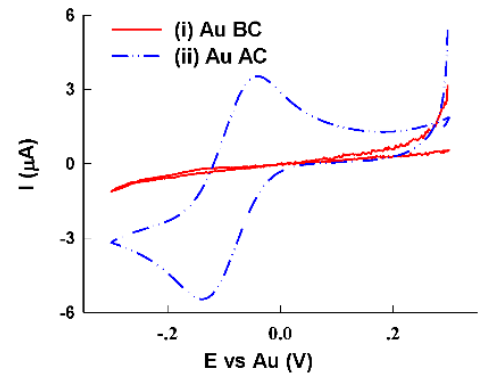

(b)
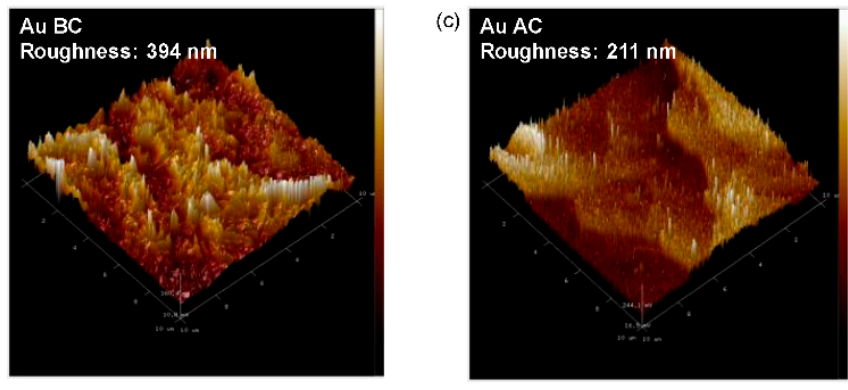

Figure 1. (a) Cyclic voltammograms obtained (at a scan rate of $50 \mathrm{mV} / \mathrm{s}$ ) in a PBS solution containing $4 \mathrm{mM} \mathrm{K}_{3} \mathrm{Fe}(\mathrm{CN})_{6}$ (i) before cleaning, (ii) after cleaning the Au plated PCB electrodes. (b) AFM of Au plated electrodes obtained (b) before cleaning, (c) after cleaning.
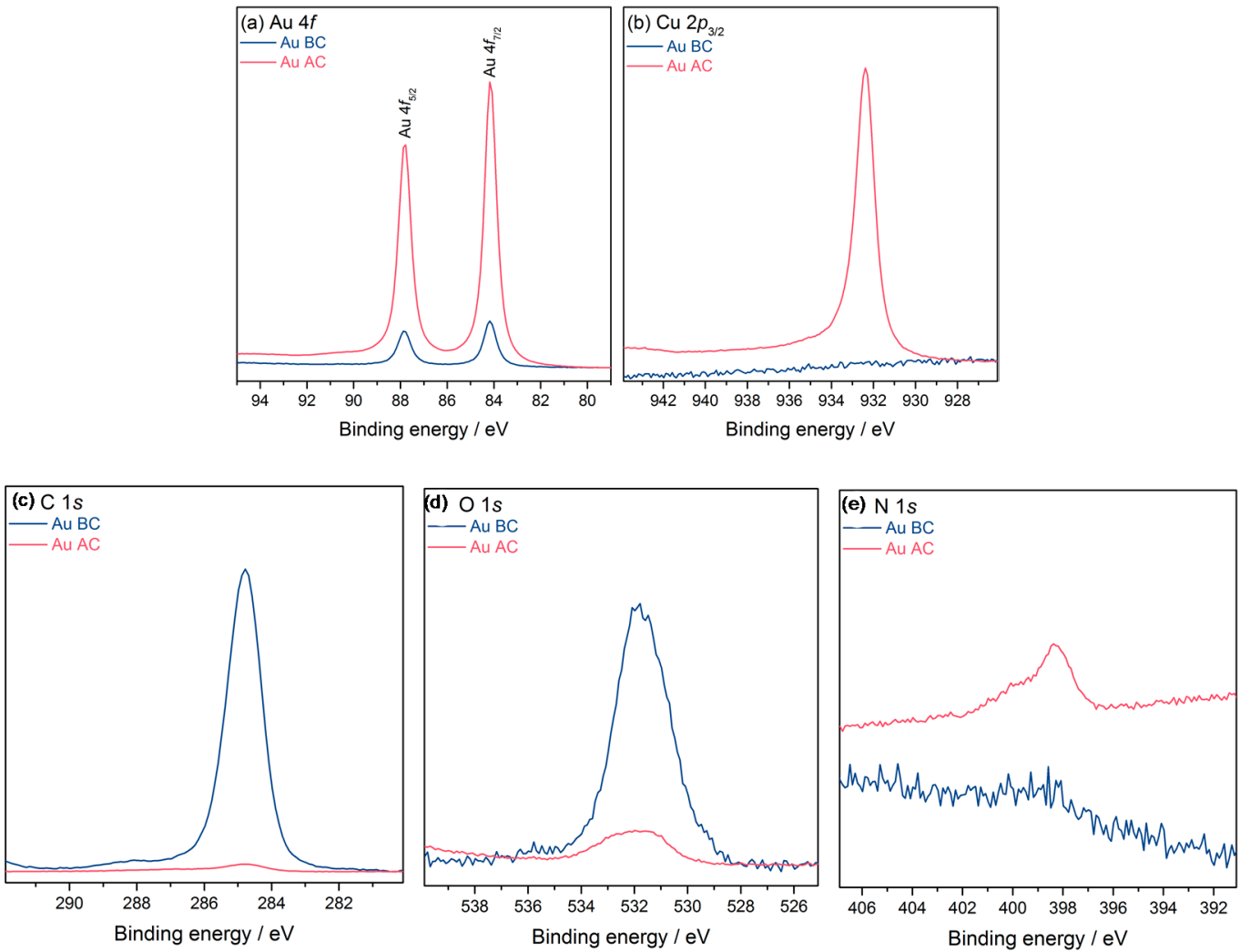

Figure 2. XPS spectra (a) Au 4f, (b) Cu 2p3/2, (c) C 1s, (d) O 1s, and (e) N 1s regions obtained before and after cleaning process of gold-plated PCB electrodes. 


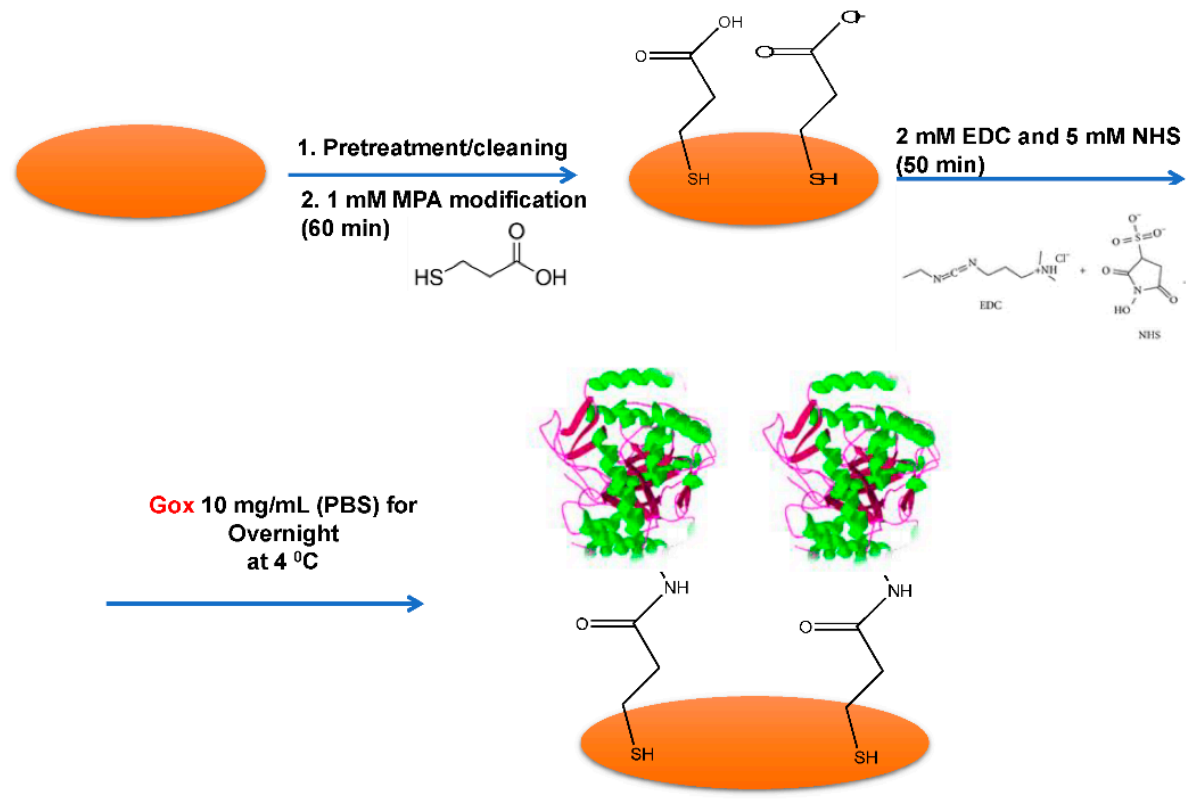

Scheme 1. Schematic illustration of the GOx immobilization on PCB surface.
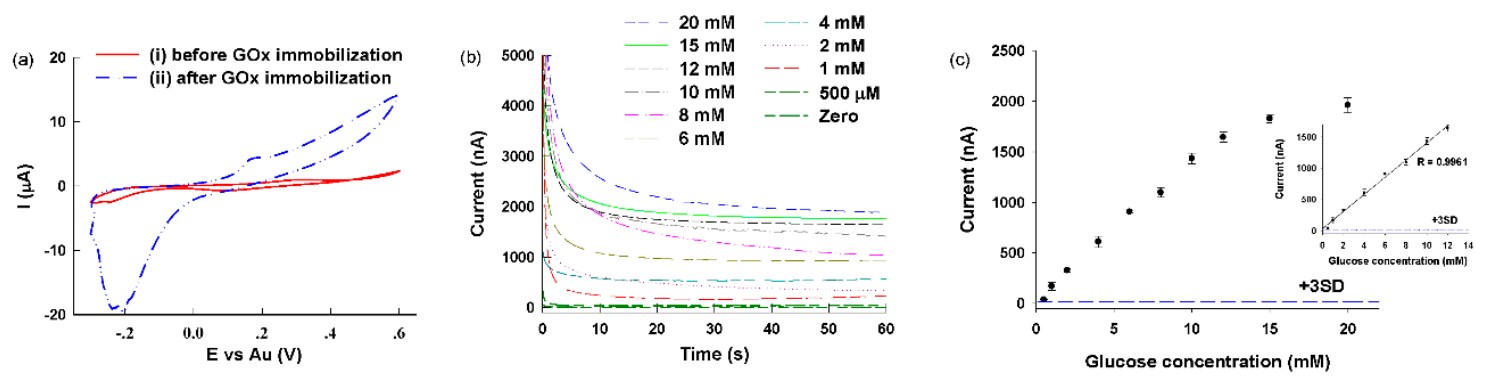

Figure 3. (a) Cyclic voltammograms obtained (at a scan rate of $50 \mathrm{mV} / \mathrm{s}$ ) in a PBS solution containing $5 \mathrm{mM}$ glucose (i) before GOx immobilization and (ii) after GOx immobilization on the Au plated PCB surface. (b) Amperometric response of GOx modified PCB biosensor with 0-20 mM glucose in PBS. (c) Calibration plot of glucose concentration based on chronoamperometric currents at $60 \mathrm{~s}$ from the response profile in panel a. Each data point represents the mean \pm SD of three separate measurements obtained using new sensors. Inset shows a magnified view of the data points at $500 \mu \mathrm{M}$ to $12 \mathrm{mM}$.

\section{Conclusions}

Our work focused on the development of a sensitive and reliable glucose biosensor, exploiting for the first time covalent immobilization of commercially fabricated PCB electrodes. Our PCBimplemented electrochemical glucose biosensor using covalently immobilized glucose oxidase (GOx) shows very good sensitivity towards glucose oxidation and could be used for point-of-care testing.

Author Contributions: G.D. and D.M. conceived the idea and designed the experiments. D.M. designed the sensing platform and microfluidics, G.D. fabricated the sensors, carried out electrochemical measurements and analyzed the data. A.R. contributed the XPS analysis. G.D. and D.M. wrote the manuscript.

Acknowledgments: The authors wish to acknowledge the financial support of British Council (Newton Fund Institutional Links, UK-Turkey project: 336872) and also thank the Spirit Circuits Group and Lyncolec Ltd for their collaboration in manufacturing the prototypes. AR acknowledges the support from Imperial College London for her Imperial College Research Fellowship.

Conflicts of Interest: The authors declare no conflict of interest. 


\section{References}

1. Singh, N.; Kesherwani, R.; Tiwari, A.K.; Kumar, D. A review on diabetes mellitus. Pharma Innov. J. 2016, 5, 36-40.

2. Available online: https://www.idf.org/our-network/regions-members/europe/members/163-turkey.html (accessed on 26 Novembe 2018).

3. Moschou, D.; Tserepi, A. The lab-on-PCB approach: Tackling the $\mu$ TAS commercial upscaling bottleneck. Lab Chip 2017, 17, 1388-1405.

4. Moschou, D.; Greathead, L.; Pantelidis, P.; Kelleher, P.; Morgan, H.; Prodromakis, T. Amperometric IFN- $\gamma$ immunosensors with commercially fabricated PCB sensing electrodes. Biosens. Bioelectron. 2016, 86, 805-810.

5. Moreira, F.T.C.; Ferreira, M.J.M.S.; Puga, J.R.T.; Sales, M.G.F. Screen-printed electrode produced by printed-circuit board technology. Application to cancer biomarker detection by means of plastic antibody as sensing material. Sens. Actuators B Chem. 2016, 223, 927-935.

6. Sanchez, J.L.; Henry, O.Y.; Joda, H.; Solnestam, B.W.; Kvastad, L.; Johansson, E.; Akan, P.; Lundeberg, J.; Lladach, N.; Ramakrishnan, D.; et al. Multiplex PCB-based electrochemical detection of cancer biomarkers using MLPA-barcode approach. Biosens. Bioelectron. 2016, 82, 224-232.

7. Salvo, P.; Henry, O.Y.; Dhaenens, K.; Acero Sanchez, J.L.; Gielen, A.; Werne Solnestam, B.; Lundeberg, J.; O'Sullivan, C.K.; Vanfleteren, J. Fabrication and functionalization of PCB gold electrodes suitable for DNAbased electrochemical sensing. Bio-Med. Mater. Eng. 2014, 24, 1705-1714.

8. Mirzajani, H.; Cheng, C.; Wu, J.; Chen, J.; Eda, S.; Aghdam, E.N.; Ghavifekr, H.B. A highly sensitive and specific capacitive aptasensor for rapid and label-free trace analysis of Bisphenol A (BPA) in canned foods. Biosens. Bioelectron. 2017, 89, 1059-1067.

9. Shanmugam, N.R.; Muthukumar, S.; Chaudhry, S.; Anguiano, J.; Prasad, S. Ultrasensitive nanostructure sensor arrays on flexible substrates for multiplexed and simultaneous electrochemical detection of a panel of cardiac biomarkers. Biosens. Bioelectron. 2017, 89, 764-772,

10. Li, X.; Zang, J.; Liu, Y.; Lu, Z.; Li, Q.; Li, C.M. Simultaneous detection of lactate and glucose by integrated printed circuit board based array sensing chip. Anal. Chim. Acta 2013, 771, 102-107.

11. Moschou, D.; Morgan, H.; Prodromakis, T. A PCB-based electrochemical glucose biosensing platform. In Proceedings of the 2016 20th International Conference on Miniaturized Systems for Chemistry and Life Sciences (microTAS 2016), Dublin, Ireland, 9-13 October 2016; pp.1047-1048.

12. Pu, Z.; Wang, R.; Wu, J.; Yu, H.; Xu, K.; Li, D. A flexible electrochemical glucose sensor with composite nanostructured surface of the working electrode. Sens. Actuators B Chem. 2016, 230, 801- 809.

13. Jamaluddin, A.; Taufik, U.; Iriani, Y.; Budiawanti, S. Suyitno Simple fabricating PCB-based inter digital capacitor for glucose biosensor. Available online: https:/aip.scitation.org/doi/abs/10.1063/1.4968345 (accessed on 28 November 2018)

(C) 2018 by the authors; Licensee MDPI, Basel, Switzerland. This article is an open access article distributed under the terms and conditions of the Creative Commons Attribution (CC BY) license (http://creativecommons.org/licenses/by/4.0/). 